\title{
Automedicação em Idosos: Medidas de Prevenção e Controle
}

\author{
Fernanda Lorena Pereira Rocha Beserra ${ }^{1}$, Valéria Ferreira da Costa Borba ${ }^{2}$, \\ Jáfia Elana Gonçalves Torres ${ }^{3}$, Sara Nayara Duarte da Silva ${ }^{4}$, \\ Mellyssa Ayêska Custodio Sobreira Macedo ${ }^{5}$
}

\begin{abstract}
RESUMO
Os medicamentos são considerados essenciais para os cuidados com a saúde, os quais colaboram para se obter uma melhora significativa no estado de saúde das pessoas, nos casos em que o utilizam de modo racional. Os idosos são os que mais consomem medicamentos sem prescrição. Diante desse cenário, o profissional farmacêutico é visto como um profissional de saúde de fácil acesso, o qual é encontrado em quase todas as farmácias do país. O objetivo deste estudo foi analisar a prevenção e o controle da automedicação no público idoso e a identificação das medidas de prevenção e controle da automedicação. Trata-se de uma Revisão Integrativa da Literatura realizada na Biblioteca Virtual de Saúde (BVS), onde foram usados os descritores indexados nos Descritores em Ciências da Saúde (Decs) "Automedicação" e "Idoso". A busca foi realizada no período de fevereiro e março de 2018, e foram encontradas 12 publicações na pesquisa, e, após os critérios de inclusão, restaram 9 trabalhos relacionados à temática. Os resultados mostraram que as principais medidas identificadas de controle e prevenção da automedicação em idosos foram a atuação da equipe de saúde, dando destaque ao farmacêutico, na divulgação de informações e esclarecimentos quanto aos riscos da automedicação por meio da adoção de práticas educativas e do uso de recurso midiático para divulgação, protocolos clínicos e listas de medicações e suas especificidades. A limitação do estudo, contudo, deu-se por um quantitativo reduzido de produções. Assim, sugerem-se estudos mais amplos que abordem essa temática, incluindo outros públicos e outros contextos.
\end{abstract}

Palavras-chave: Idoso. Automedicação. Prevenção e controle.

\section{AUTOMEDICATION IN ELDERLY: PREVENTION AND CONTROL MEASURES}

\begin{abstract}
Medicines are considered essential for health care, which help to achieve a significant improvement in people's health when used in a rational way. The elderly are the ones who consume the most drugs without prescription. Given this scenario, the pharmaceutical professional is seen as being an easily accessible health professional, which is found in almost every pharmacy in the country. The objective of this study was to analyze the prevention and control of self-medication in the elderly and the identification of measures of prevention and control of self-medication. It is an Integrative Review of the Literature carried out in the Virtual Health Library (VHL), where the descriptors indexed in the Descriptors in Health Sciences (Decs) "Self-medication" and "Elderly" were used. The search was carried out in February and March of 2018, and twelve publications were found in the research, where after the inclusion criteria there were nine papers related to the theme. The results showed that the main measures identified for controlling and preventing self-medication in the elderly were the health team, emphasizing the pharmacist, in the dissemination of information and clarification regarding the risks of self-medication through the adoption of educational practices and the use of media resources for dissemination, clinical protocols and lists of medications and their specificities. However, the limitation of the study was due to a reduced quantitative of productions, thus, it is suggested broader studies that approach this subject, including other public and other contexts.
\end{abstract}

Keywords: Old man. Self-medication. Prevention and control.

\footnotetext{
Farmacêutica graduada pela Faculdade de Juazeiro do Norte, Juazeiro do Norte-CE. fernandarocha_ri@yahoo.com.br

2 Farmacêutica. Mestre em Ciências Farmacêuticas pela Universidade Federal de Pernambuco, Recife-PE. valeria.borba@hotmail.com

3 Farmacêutica graduada pela Faculdade de Juazeiro do Norte, Juazeiro do Norte-CE. jafiagoncalves@gmail.com

4 Farmacêutica graduada pela Faculdade de Juazeiro do Norte, Juazeiro do Norte-CE. sara_duarte@outlook.com

5 Farmacêutica graduada pela Faculdade de Juazeiro do Norte, Juazeiro do Norte-CE. mellyssaayeska@hotmail.com
} 


\section{INTRODUÇÃO}

Os medicamentos são instrumentos considerados essenciais para os cuidados com a saúde, os quais colaboram para se obter uma melhora significativa no estado de saúde das pessoas, nos casos em que o utilizam de modo racional (SILVA JUNIOR; NASCIMENTO, 2016).

De acordo com o Ministério da Saúde e a Organização Mundial de Saúde (OMS), no Brasil existem mais de 32 mil medicamentos, com algumas classes sendo de venda livre, ou seja, sem necessidade de prescrição, enquanto para determinadas classes a prescrição médica é indispensável. Muitas dessas classes de medicamentos, entretanto, são comercializados de modo indiscriminado por alguns estabelecimentos farmacêuticos. Para a população, em geral, mediante a dificuldade de acesso encontrada na saúde pública no Brasil, as farmácias passaram a apresentar-se, muitas vezes, como o primeiro recurso para solucionar problemas de saúde e alcançar o bem-estar (NASCIMENTO; VALDÃO, 2012).

Neste contexto, a automedicação torna-se presente. Esta é caracterizada quando acontece a utilização de medicamentos para o tratamento de doenças e/ou sintomas que são percebidos pelas pessoas, sem prescrição ou orientação de um profissional prescritor habilitado, cabendo ao próprio paciente a decisão de qual medicamento ele irá utilizar (VERNIZI; SILVA, 2016).

Entre todas as faixas etárias, os idosos são os que consomem uma quantidade maior de medicamentos sem prescrição, talvez pelo fato de serem os que mais sofrem com doenças quando comparados com outras faixas etárias ou por fazerem a utilização, em média, de dois a cinco fármacos diariamente, tornando-os mais sensíveis a efeitos adversos e a interações medicamentosas. Com isso, os idosos tornam-se os mais suscetíveis à automedicação (PEREIRA et al., 2017; BEZERRA; BRITO; COSTA, 2016).

Diante desse cenário, o profissional farmacêutico é visto como um profissional de saúde de fácil acesso, o qual é encontrado em quase todas as farmácias do país, e a sua atuação pode contribuir para a melhoria da população, posto que a automedicação é uma conduta bastante comum Brasil. $O$ fato de a população idosa realizar a automedicação, sem critérios técnicos e acompanhamento de um profissional qualificado, caracteriza essa prática como sendo de utilização irracional de medicamentos (FERNANDES; CEMBRANELLI, 2015).

Este estudo teve como objetivo identificar as medidas de prevenção e controle da automedicação no público idoso.

\section{METODOLOGIA}

Trata-se de um estudo de revisão integrativa de literatura acerca da automedicação em idosos.

A presente pesquisa foi realizada por meio do levantamento bibliográfico disponível na Biblioteca Virtual de Saúde (BVS), que abordasse a temática "prevenção e controle da automedicação no público idoso". Para o alcance do objetivo proposto estabelecemos como pergunta norteadora para a revisão: "Quais as medidas de prevenção e controle da automedicação no público idoso?"

A pesquisa foi realizada em fevereiro e março de 2018, e abrangeu as seguintes etapas: definição das palavras-chave; definição dos critérios de inclusão para a seleção; busca dos estudos selecionados para a análise; seleção e análise dos trabalhos pertinentes; interpretação dos resultados e síntese da revisão.

Os critérios de inclusão definidos para a seleção dos artigos foram: publicações em língua portuguesa, limitando-se a textos gratuitos, disponíveis na íntegra e de modo on-line que tivessem relação com o tema proposto.

As bases de dados utilizadas foram: Base de Dados de Enfermagem (BDENF), Literatura Latino Americana e do Caribe (Lilacs) e a Literatura Internacional em Ciências da Saúde (Medline), com recorte temporal no período de 2013 a 2017. Os descritores utilizados foram: "Automedicação" e "Idoso", empregando o boleador "AND", baseados nos Descritores em Ciências da Saúde (Decs).

\section{RESULTADOS E DISCUSSÃO}

\section{Caracterização dos Estudos}

Utilizando o descritor "Automedicação" foram encontradas 66 publicações: 45 na base de dados Lilacs, 11 na Medline e 12 na BDENF. Refinando a busca, foi adicionado o descritor "Idoso", o que resultou em 12 trabaIhos, sendo 6 na Lilacs, 2 na BDENF e 4 na Medline.

Destes 12 textos, apenas 9 atenderam aos critérios de inclusão e 2 foram excluídos por estarem duplicados. Foram realizadas as análises do título, dos descritores e do resumo dos 10 textos publicados. Nessa etapa, 1 trabalho foi excluído por não ter relação com a temática proposta deste trabalho. Nove trabalhos, portanto, foram incluídos, pois estavam relacionados à temática, ao objetivo deste estudo e contemplavam os critérios de inclusão preestabelecidos. As principais conclusões destes trabalhos selecionados estão descritas na Tabela 1. 
Tabela 1 - Distribuição das referências incluídas no estudo, de acordo com o ano de publicação, título, base de dados, periódico e objetivos propostos

\begin{tabular}{|c|c|c|}
\hline AUTORES(ANO) & $\begin{array}{l}\text { BASE DE } \\
\text { DADOS }\end{array}$ & PERIÓDICO \\
\hline
\end{tabular}

\begin{tabular}{llll}
\hline $\begin{array}{l}\text { SANTOS et al. } \\
\text { (2013) }\end{array}$ & Lilacs & $\begin{array}{l}\text { Revista de Saúde } \\
\text { Pública }\end{array}$ & $\begin{array}{l}\text { Analisar o padrão de consumo } \\
\text { de medicamentos entre } \\
\text { idosos e sua associação com } \\
\text { aspectos socioeconômicos e sua } \\
\text { autopercepção. }\end{array}$ \\
$\begin{array}{l}\text { NEVES et al. } \\
\text { (2013) }\end{array}$ & Lilacs & $\begin{array}{l}\text { Revista de Saúde } \\
\text { Pública }\end{array}$ & $\begin{array}{l}\text { Analisar o uso de medicamentos } \\
\text { entre idosos e os fatores associados. }\end{array}$
\end{tabular}

As práticas da polifarmácia e automedicação e uso inapropriado de medicamentos foram equivalentes as demais regiões do país. entre idosos e os fatores associados.

A proporção do uso excessivo de medicamentos demostrou diferenças em relação à escolaridade, quantidade de consultas médicas e saúde autorreferida.

\begin{tabular}{llll}
$\begin{array}{l}\text { BARROSO et al. } \\
\text { (2017) }\end{array}$ & BDENF & $\begin{array}{l}\text { Revista de } \\
\text { Enfermagem } \\
\text { UFPE }\end{array}$ & $\begin{array}{l}\text { Verificar a prevalência da } \\
\text { automedicação de idosos, bem como } \\
\text { identificar os grupos terapêuticos dos } \\
\text { medicamentos autoadministrados. }\end{array}$ \\
\hline ELY et al. (2015) Lilacs & $\begin{array}{l}\text { Revista Brasileira } \\
\text { de Geriatria e } \\
\text { Gerontologia }\end{array}$ & $\begin{array}{l}\text { Analisar a prevalência do uso de anti- } \\
\text { inflamatórios e analgésicos em idosos } \\
\text { da Estratégia Saúde da Família de } \\
\text { Porto Alegre-RS. }\end{array}$
\end{tabular}

A automedicação configura-se um problema de saúde pública em razão da alta prevalência e necessidade de ações em saúde voltadas para o perfil dos praticantes.

A falta de autopercepção de saúde propicia ao uso indevido do medicamento. Os profissionais de saúde devem ter um olhar mais individual para melhor orientar o idoso.

\begin{tabular}{|c|c|c|c|c|}
\hline $\begin{array}{l}\text { GOULART et al. } \\
(2014)\end{array}$ & Lilacs & $\begin{array}{l}\text { Estudos } \\
\text { interdisciplinares } \\
\text { sobre o } \\
\text { envelhecimento }\end{array}$ & $\begin{array}{l}\text { Avaliar o consumo de medicamentos } \\
\text { por idosos usuários da Unidade } \\
\text { Básica de Saúde Dom Bosco do } \\
\text { município de Rondonópolis/MT. }\end{array}$ & $\begin{array}{l}\text { Acompanhar a farmacoterapia do } \\
\text { idoso é fundamental, promovendo } \\
\text { o uso racional dos medicamentos } \\
\text { tornando-o mais instruído. }\end{array}$ \\
\hline $\begin{array}{l}\text { TELLES FILHO; } \\
\text { ALMEIDA; } \\
\text { PINHEIRO } \\
(2013)\end{array}$ & BDENF & $\begin{array}{l}\text { Revista } \\
\text { Enfermagem Uerj }\end{array}$ & $\begin{array}{l}\text { Descrever a automedicação no que } \\
\text { concerne à frequência, motivos, } \\
\text { justificativas, tempo de ingesta e } \\
\text { influências, em idosos pertencentes } \\
\text { a uma Estratégia Saúde da Família de } \\
\text { um município do interior do Estado } \\
\text { de Minas Gerais }\end{array}$ & $\begin{array}{l}\text { O desenvolvimento de ações } \\
\text { educativas em saúde devem ser } \\
\text { uma prioridade, uma vez que a } \\
\text { automedicação está intimamente } \\
\text { relacionada à falta de informações. }\end{array}$ \\
\hline $\begin{array}{l}\text { LUTZ; } \\
\text { MIRANDA; } \\
\text { BERTOLDI } \\
\text { (2017) }\end{array}$ & Medline & $\begin{array}{l}\text { Revista de Saúde } \\
\text { Pública }\end{array}$ & $\begin{array}{l}\text { Avaliar o uso de medicamentos } \\
\text { potencialmente inadequados entre } \\
\text { idosos. }\end{array}$ & $\begin{array}{l}\text { Tornar o idoso consciente dos } \\
\text { riscos do uso inapropriado de } \\
\text { medicamentos com o auxílio de } \\
\text { listas mais especificas para esse } \\
\text { público na Rename. }\end{array}$ \\
\hline $\begin{array}{l}\text { BEZERRA; } \\
\text { BRITO; COSTA } \\
\text { (2016) }\end{array}$ & Medline & $\begin{array}{l}\text { Revista Cogitare } \\
\text { Enfermagem }\end{array}$ & $\begin{array}{l}\text { Caracterizar o uso de medicamentos } \\
\text { entre idosos atendidos em uma } \\
\text { Unidade Básica de Saúde da Família. }\end{array}$ & $\begin{array}{l}\text { Práticas de administração dos } \\
\text { fármacos e conhecer suas } \\
\text { características é essencial para a } \\
\text { orientação adequada ao idoso. }\end{array}$ \\
\hline $\begin{array}{l}\text { ARAÚJO- } \\
\text { JÚNIOR; } \\
\text { VICENTINI } \\
(2007)\end{array}$ & Lilacs & $\begin{array}{l}\text { Arquivos de } \\
\text { Ciências da Saúde } \\
\text { da Unipar }\end{array}$ & $\begin{array}{l}\text { Promover um levantamento } \\
\text { da prevalência da prática da } \\
\text { automedicação e traçar suas } \\
\text { características na cidade de Guairaçá- } \\
\text { PR }\end{array}$ & $\begin{array}{l}\text { O uso de medicamentos sem } \\
\text { prescrição é uma prática de difícil } \\
\text { controle, tornando-se fundamental } \\
\text { otimizar a qualidade da atenção } \\
\text { farmacêutica e médica. }\end{array}$ \\
\hline
\end{tabular}


No que se refere à caracterização dos trabaIhos segundo o ano de publicação, observa-se que os primeiros, conforme os critérios preestabelecidos, datam do início no ano de 2007. Nos anos seguintes (2008, 2009, 2010 e 2011) não foram evidenciadas publicações. Em 2012 foi encontrada uma publicação. Já em 2013 duas publicações foram encontradas. Em 2014, 2015 e 2016 foi contatada uma publicação em relação a cada ano. Por fim, em 2017 teve-se duas publicações no ano.

A maior incidência de publicação deu-se na base de dados da Lilacs com cinco estudos, seguido da Medline e BDENF com dois cada. No que se refere aos objetivos, a maioria dos estudos selecionados procura identificar a prevalência da prática de automedicação.

\section{Medidas de Prevenção e Controle da Automedicação no Público Idoso}

A utilização de medicamentos entre os idosos configura-se como uma importante estratégia terapêutica com o propósito de equilibrar as alterações advindas do processo de envelhecimento. Assim, "o acompanhamento farmacoterapêutico do idoso é fundamental para a promoção do uso racional de medicamentos, visando a contribuir no processo educativo dos usuários acerca do conhecimento da sua terapia medicamentosa" (GOULART et al., 2014)

$O$ entendimento a respeito do consumo de medicamentos pelos idosos e os fatores relacionados é indispensável para as redefinições das políticas públicas, na procura de melhorias das condições de vida e de saúde dos idosos (SANTOS et al., 2013).

Ao ter acesso aos principais fatores, dados e outras informações referentes ao consumo de fármacos de forma indiscriminada por idosos, é possível que os gestores atuantes possam programar suas ações, indo ao encontro da realidade local. Dessa forma, podem-se compreender quais medidas de controle e prevenção são mais adequadas e podem surtir melhores efeitos.

Faz-se necessária a colaboração dos profissionais de saúde no que refere à utilização de modo racional dos medicamentos pela população idosa, com a finalidade de reduzir as complicações provenientes do seu consumo. Além disso, deve-se alertar os gestores em saúde para adaptar o sistema de assistência à saúde para a real demanda dos idosos existentes, assim como em preparar os futuros idosos (SANTOS et al., 2013).

Esse cenário deixa evidente a necessidade de realizar-se campanhas para informar e conscientizar a população no que se refere ao uso correto dos medi- camentos disponíveis no mercado, quando se faz indispensável a participação dos profissionais de saúde, em especial os médicos e farmacêuticos (ARAÚJO JÚNIOR; VICENTINI, 2007).

O trabalho coletivo em prol da redução dos índices referentes à automedicação em idosos mostra-se relevante. Os profissionais necessitam de conhecimentos científicos adequados para informar sobre os riscos e possíveis consequências da automedicação, esclarecendo dúvidas e reforçando a importância de somente utilizar fármacos quando prescritos por profissionais devidamente habilitados, respeitando a posologia.

Desse modo, o farmacêutico torna-se essencial na educação e no incentivo sobre a utilização de modo racional dos medicamentos, deixando evidente a necessidade de que esse profissional de saúde possua a consciência de alertar os idosos a respeito dos riscos provenientes do consumo de fármacos sem a orientação adequada (ARAÚJO JÚNIOR; VICENTINI, 2007).

Ressalta-se, ainda, que é atribuição do farmacêutico o fornecimento de orientações sobre os medicamentos com o propósito de reduzir os riscos e proporcionar a maior eficácia possível, promovendo, desse modo, a educação em saúde para as precisões dos idosos, levando em consideração as práticas que podem causar danos à saúde, assim como na promoção de reflexões e discussões sobre a temática para toda a população, abrangendo também os demais profissionais da saúde, gestores e políticos (TELLES FILHO; ALMEIDA; PINHEIRO, 2013).

Destaca-se a figura do farmacêutico por este ter contato durante sua formação com os fármacos e seus principais aspectos, grande propriedade no que se refere a aspectos científicos. Ressalta-se, entretanto, que o mesmo deve atuar no combate à automedicação, observando a necessidade de uma prescrição e respeitando a quantidade necessária para a terapia proposta.

O acompanhamento farmacoterapêutico dos idosos é um passo essencial para a promoção da utilização correta dos medicamentos, e, por meio da abordagem educativa, é possível a colaboração entre os profissionais de saúde, o que favorecerá o esclarecimento das dúvidas, redução da ansiedade e proporcionará uma maior eficácia no emprego de medidas terapêuticas (GOULART et al., 2014). 
Destaca-se, ainda, a necessidade de conscientizar de modo crítico os profissionais da saúde, de maneira que possam ser multiplicadores das informações sobre a utilização racional dos medicamentos (TELLES FILHO; ALMEIDA; PINHEIRO, 2013).

Uma abordagem educativa de conscientização fortalece um discurso horizontal, quando clientes e profissionais compartilham de informações e experiências e compreendem, de maneira integrada, o melhor caminho a se seguir. No caso da automedicação em pacientes idosos, o uso de práticas educativas mostra-se como um importante mecanismo de prevenção e controle dessa prática, por proporcionar a divulgação de informações de maneira mais significativa e adequar-se à linguagem necessária para se fazer entendido.

É necessária ampla discussão sobre a necessidade de adoção de medidas para a promoção do uso racional de medicamentos entre essa parcela da população, educação continuada dos profissionais prescritores, qualificação dos sistemas de saúde para oferecer educação permanente e acesso a informações adequadas em momento oportuno, e adoção de medidas no âmbito da assistência farmacêutica por meio da elaboração e implementação de listas de medicamentos e protocolos clínicos adequados às necessidades da população idosa (NEVES et al., 2013, p. 4).

Os profissionais atuantes, seja em esfera pública ou privada, devem buscar constantemente atualizações. As empresas e órgãos também devem ofertar capacitações e oficinas que permitam qualificar seu quadro profissional. Ao instrumentar um profissional com conhecimento direcionado à questão em estudo, o mesmo pode pôr em prática ações de controle e prevenção à automedicação, tornando seu espaço de trabalho um ambiente de promoção e divulgação de informações.

É importante o desenvolvimento de critérios para as prescrições no Brasil, as quais devem contemplar todos os fármacos disponíveis no país. Além disso, é preciso que a Relação Nacional de Medicamentos Essenciais (Rename) contenha os medicamentos para utilização em idosos, assim como na ampliação da disponibilidade para os usuários do Sistema Único de Saúde (SUS). "Outra recomendação é a maior ênfase nos currículos das faculdades sobre as especificidades do uso de medicamentos entre idosos, informando os futuros profissionais sobre prescrições potencialmente inadequadas para essa faixa etária" (LUTZ; MIRANDA; BERTOLDI, 2017).
A relação de medicamentos potencialmente inapropriados para idosos mais citadas e utilizadas são os Critérios de Beers, desenvolvidos nos Estados Unidos em 1991, e o Screening Tool of Older Persons' Potentially Inappropriate Prescriptions (STOPP), elaborado na Irlanda, em 2008. Ambos foram desenvolvidos por meio do consenso de uma equipe de especialistas nacionais, composta, dentre outros, por geriatras, farmacologistas e farmacêuticos clínicos, utilizando a técnica Delphi (BERRS et al., 1991; GALLAGHER; O'MAHONY, 2008).

Tanto os Critérios de Beers quanto o STOPP foram submetidos a revisões com abordagem baseada em evidências, e suas versões mais recentes constam listas de medicamentos/grupos farmacológicos que devem ser evitados em todos os idosos, aqueles que devem ser evitados em idosos com determinada condição clínica e os que devem ser utilizados com precaução (AMERICAN GERIATRICS SOCIETY, 2012, 2015, 2019; O'MAHONY et al., 2015).

Os profissionais devem começar a ter contato com esta temática já no nível da Graduação, preparando-se para atuar em situações específicas, recebendo o suporte teórico necessário para a construção de competências na sua área de atuação.

A identificação das características e dos fatores relacionado à utilização de medicamentos pelos idosos auxilia na idealização das medidas voltadas para a promoção do consumo de modo racional dos fármacos, quando, dessa maneira, ocorrerá uma melhoria na qualidade de vida dos mesmos, bem como na contribuição para a redução dos gastos desnecessários (ELY et al., 2015).

É indispensável que seja feita a identificação e a análise da automedicação nos idosos, pois essa informação pode ser a base para estratégias de saúde, "possibilitando o planejamento do uso racional de medicamentos, fornecendo subsídios para a maximização das condições de saúde individual e coletiva, bem como para a realização de projetos de cunho preventivo e/ou curativo" (TELLES FILHO; ALMEIDA; PINHEIRO, 2013, p. 5).

O diagnóstico situacional de qualquer problema de saúde pública mostra-se fundamental. Os profissionais necessitam compreender os aspectos do público em questão, seu perfil, os fatores que os levam à ingestão de medicamentos de forma indiscriminada e a percepção dos mesmos sobre os riscos existentes; apenas assim pode-se planejar ações direcionadas, que consideram o contexto que perpassa a automedicação em pacientes idosos. 
Fica evidente a necessidade de direcionar as medidas de educação em saúde a respeito do uso de medicamentos pelos idosos, com o a finalidade de reduzir os prejuízos originados pela automedicação (BARROSO et al., 2017; ARAÚJO JÚNIOR; VICENTINI, 2007).

O uso de recursos de mídia pode ser um incremento no controle e prevenção da automedicação. Utilizar meios que se adequem ao perfil do público em questão é crucial, posto que se deve levar em consideração que certas mídias podem ser de difícil acesso aos mesmos ou, ainda, que não tragam as informações de maneira clara e objetiva.

É necessário que sejam desenvolvidas atividades de educação em saúde, focando nos pontos negativos da automedicação, com o propósito de abranger os familiares que indicam a utilização de medicamentos sem prescrição médica. Desse modo, essas atitudes possuem um reflexo de médio e longo prazos, com uma redução na realização da automedicação. Assim, "destaca-se a necessidade da inclusão da família nos diferentes momentos do processo educativo do paciente por meio de participação ativa, visando à ampliação do comprometimento com os pacientes" (TELLES FILHO; ALMEIDA; PINHEIRO, 2013, p. 2).

Os familiares e cuidadores devem ser aliados no processo de controle e prevenção da automedicação. Por estarem em contato contínuo com os idosos, esses podem atuar na supervisão e instrução dos mesmos, orientando e supervisionando, quando necessário, o uso da medicação. Para que isso ocorra os profissionais devem incluir os familiares e cuidadores no processo de cuidar, respeitando suas limitações e particularidades.

A preocupação com a saúde do idoso e a sua qualidade de vida não é restrita somente à sua família; é preciso uma discussão a respeito da criação de uma rede de apoio para assistência ao idoso com um enfoque multidimensional, levando em consideração as questões psicológicas, as relações sociais e o meio ambiente (TELLES FILHO; ALMEIDA; PINHEIRO, 2013).

A abordagem ao idoso adepto à automedicação deve ir além da estrutura física dos consultórios. É preciso refletir sobre maneiras de integrá-lo a outras atividades que contribuam com sua qualidade de vida, ofertando uma assistência holística e humanizada por parte de todos os profissionais envolvidos no seu processo saúde-doença.

\section{CONSIDERAÇÕES FINAIS}

A terapia farmacológica é uma importante aliada no controle e combate de patologias. Os fármacos atuam em prol da melhoria do quadro clínico do pa- ciente, todavia torna-se relevante compreender que o uso indiscriminado dos mesmos por pacientes idosos representa um fator de risco para desenvolvimentos de outras patologias e alterações gerais.

As principais medidas identificadas de controle e prevenção da automedicação em idosos foram a atuação da equipe de saúde, dando destaque ao farmacêutico, na divulgação de informações e esclarecimentos quanto aos riscos da automedicação por meio da adoção de práticas educativas, e o uso de recurso midiático para divulgação, protocolos clínicos e listas de medicações e suas especificidades. Constatou-se, ainda, que a inclusão da família e cuidadores como potencializadores desse controle e prevenção também se mostrou relevante.

A limitação do estudo deu-se por um quantitativo reduzido de produções; assim, sugerem-se estudos mais amplos que abordem essa temática, incluindo outros públicos e outros contextos.

\section{REFERÊNCIAS}

AMERICAN GERIATRICS SOCIETY. Updated Beers Criteria for Potentially Inappropriate Medication use in Older Adults. Journal of the American Geriatrics Society, v. 60, n. 4, p. 616-631, 2012. Available from: https://onlinelibrary.wiley. com/doi/abs/10.1111/j.1532-5415.2012.03923.x. Cited: 10 June 2019.

AMERICAN GERIATRICS SOCIETY. Updated Beers Criteria for Potentially Inappropriate Medication use in Older Adults. Journal of the American Geriatrics Society, v. 63, n. 11, p. 2.227-2.246, 2015. Available from: https://www.sigot. org/allegato_docs/1057_Beers-Criteria.pdf. Cited: 10 June 2019.

AMERICAN GERIATRICS SOCIETY. Updated AGS Beers Criteria $^{\circledR}$ for Potentially Inappropriate Medication use in Older Adults. Journal of the American Geriatrics Society, v. 67, n. 4, p. 674-694, 2019. Available from: https://onlinelibrary. wiley.com/doi/10.1111/jgs.15767. Cited: 10 June 2019.

ARAÚJO-JÚNIOR, J. C.; VICENTINI, G. E. Automedicação em adultos na cidade de Guairaçá - PR. Arquivos de Ciências da Saúde Unipar, Umuarama, v. 11, n. 2, p. 83-88, maio/ago. 2007. Disponível em: revistas.unipar.br/index.php/saude/ article/view/1518/1333. Acesso em: 5 mar. 2018.

BARROSO, R. et al. Automedicação em idosos de estratégias de saúde da família. Revista de Enfermagem da Ufpe, v. 11, n. 2, fev. 2017. Disponível em: bases.bireme.br/cgi-bin/wxislind.exe/iah/online/?IsisScript=iah/iah.xis\&src=google\&base=BDENF\&lang $=p$ \&nextAction=Ink\&exprSear $\mathrm{ch}=30860$ \&indexSearch=ID. Acesso em: 5 mar. 2018.

BEZERRA, T. A.; BRITO, M. A. A.; COSTA, K. N. F. M. Caracterização do uso de medicamentos entre idosos atendidos em uma Unidade Básica de Saúde da família. Revista Cogitare 
Enfermagem, v. 21, n. 1, p. 1-11, jan./mar. 2016. Disponível em: http://revistas.ufpr.br/cogitare/article/view/43011. Acesso em: 10 fev. 2018.

BEERS, M. H. et al. Explicit criteria for determining inappropriate medication use in nursing home residents. UCLA Division of Geriatric Medicine. Archives of Internal Medicine, v. 151, n. 9, p. 1.825-1.832. 1991. Available from: https:// jamanetwork.com/journals/jamainternalmedicine /article-abstract/615518. Cited: 10 June 2019.

ELY, L. S. et al. Uso de anti-inflamatórios e analgésicos por uma população de idosos atendida na Estratégia Saúde da Família. Revista Brasileira de Geriatria e Gerontologia, v. 18, n. 3, p. 475-485, 2015. Disponível em: www.scielo.br/scielo. php?pid=S1809-98232015000300475\&script=sci_abstract\&tlng=pt. Acesso em: 5 mar. 2018.

FERNANDES, W. S.; CEMBRANELLI, J. C. Automedicação e o uso irracional de medicamentos: o papel do profissional farmacêutico no combate a essas práticas. Revista Univap, São José dos Campos, v. 21, n. 37, jul. 2015. Disponível em: revista.univap.br/index.php/revistaunivap/article/viewFile/265/259. Acesso em: 10 fev. 2018.

GALLAGHER, P.; O'MAHONY, D. Stopp (Screening Tool of Older Persons' potentially inappropriate Prescriptions): application to acutely ill elderly patients and comparison with Beers' criteria. Age and Ageing, v. 37, n. 6, p. 673-679, 2008. Disponível em: https://academic.oup.com/ageing/article/37/6/673/40813. Acesso em: 10 jun. 2019.

GOULART, L. S. et al. Consumo de medicamentos por idosos de uma Unidade Básica de Saúde de Rondonópolis/MT. Revista de Estudos Interdisciplinares Sobre o Envelhecimento, v. 19, n. 1, 2014. Disponível em: seer.ufrgs.br/index.php/RevEnvelhecer/article/view/25854. Acesso em: 5 mar. 2018.

LUTZ, B. H.; MIRANDA, V. I.; BERTOLDI, A. D. Inadequação do uso de medicamentos entre idosos em Pelotas, RS. Revista de Saúde Pública, v. 51, n. 52, 2017. Disponível em: www.scielo.br/pdf/rsp/v51/pt_0034-8910-rsp-S1518-87872017051006556.pdf. Acesso em: 5 mar. 2018.

MOHER, D. et al. Principais itens para relatar revisões sistemáticas e meta-análises: a recomendação Prisma. Revista Epidemiologia a Serviço da Saúde, Brasília, v. 24, n. 2, abr./ jun. 2015. Disponível em: www.scielo.br/pdf/ress/v24n2/ 2237-9622-ress-24-02-00335.pdf. Acesso em: 25 jan. 2018.
NASCIMENTO, J. P.; VALDÃO, G. B. M. Automedicação: educação para prevenção. In: CIEGESI, 1.; ENCONTRO CIENTÍFICO DO PNAP, 1., 2012. Disponível em: http://www.anais. ueg.br/index.php/ciegesi/article/view/1173. Acesso em: 10 fev. 2018

NEVES, S. J. F. et al. Epidemiologia do uso de medicamentos entre idosos em área urbana do Nordeste do Brasil. Revista de Saúde Pública, v. 47, n. 4, 2013. Disponível em: www.scielo.br/scielo. php?pid=S0034=89102013000400759-\&scriptsci_abstract\&tlng=pt. Acesso em: 5 mar. 2018.

O'MAHONY, D. et al. STOPP/START criteria for potentially inappropriate prescribing in older people: version 2. Age and Ageing, v. 44, n. 2, p. 213-218, 2015. Available from: https:// academic.oup.com/ageing/article/44/2/213/2812233. Cited: 10 jun. 2019.

PEREIRA, F. G. F. et al. Automedicação em idosos ativos. Revista de Enfermagem da Ufpe, Recife, v. 11, n. 12, p. 4.9194.928, dez. 2017. Disponível em: https://periodicos.ufpe.br/ revistas/revistaenfermagem/article/viewFile/22289/25307. Acesso em: 10 fev. 2018.

SANTOS, T. R. A. et al. Consumo de medicamentos por idosos, Goiânia, Brasil. Revista de Saúde Pública, São Paulo, v. 47, n. 1, fev. 2013. Disponível em: www.scielo.br/scielo. php?script=sci_arttext $\&$ pid=S0034-89102013000100013. Acesso em: 5 mar. 2018.

SILVA JUNIOR, D. N.; NASCIMENTO, E. G. C. Prática da automedicação entre os idosos: uma revisão integrativa da literatura. In: CONGRESSO NACIONAL DE ENVELHECIMENTO HUMANO, 1., 2016. Disponível em: www.editorarealize. com.br/revistas/cneh/trabalhos/TRABALHO_EV054_MD2_ SA3_ID590_10092016182846.pdf. Acesso em: 10 fev. 2018.

TELLES FILHO, P. C. P.; ALMEIDA, A. G. P.; PINHEIRO, M. L. P. Automedicação em idosos: um problema de saúde pública. Revista de Enfermagem da Uerj, Rio de Janeiro, v. 21, n. 2, p. 197-201, abr./jun. 2013. Disponível em: www.facenf.uerj. br/v21n2/v21n2a10.pdf. Acesso em: 5 mar. 2018.

VERNIZI, M. D.; SILVA, L. L. A prática de automedicação em adultos e idosos: uma revisão de literatura. Revista Saúde e Desenvolvimento, v. 10, n. 5, jul./dez. 2016. Disponível em: https://www.uninter.com/revistasaude/index.php/saudeDesenvolvimento/article/viewFile/579/345. Acesso em: 10 fev. 2018. 